\section{Die Darstellung von Menschen mit Autismus-Spektrum-Störung in Romanen - Eine Analyse mit Bezug auf Lebenszufriedenheit}

\section{Carmen Feldhaus}

Carl von Ossietzky Universität Oldenburg

Bei diesem Forschungsprojekt handelt es sich um das laufende Habilitationsvorhaben von Dr. Carmen Feldhaus am Lehrstuhl Pädagogik bei Beeinträchtigung der emotionalen und sozialen Entwicklung der Fakultät für Bildungs- und Sozialwissenschaften der Carl von Ossietzky Universität Oldenburg.

\section{Hintergrund}

In den letzten Jahren ist eine Zunahme der Anzahl von Personen mit der Diagnose AutismusSpektrum-Störung zu verzeichnen. Es ist unklar, womit der Anstieg genau zusammenhängt: Zum einen gab es Änderungen in den Störungsbildbeschreibungen der Klassifikationssysteme DSM und ICD, zum anderen achten Eltern genauer darauf, ob sich ihr Kind "normal“ entwickelt. Der Bekanntheitsgrad der Autismus-Spektrum-Störung ist innerhalb der Bevölkerung gestiegen, ein Umstand, der auch mit den Medien zusammenhängt. Ein Film wie der bekannte "Rain Man“, welcher vor 35 Jahren noch Seltenheitswert hatte und einen Hauptdarsteller zeigte, der an einer schweren Entwicklungsstörung litt, ist heutzutage nichts Ungewöhnliches mehr. So gibt es inzwischen zahlreiche Kino- und Fernsehfilme, in denen Autismus eine Rolle spielt. Das jüngste Beispiel ist der Tatort "Schwanensee“ von Anfang November 2015: In einem Schwimmbecken liegt die Leiche einer Patientin des Therapiezentrums Schwanensee. Ein Mann schwimmt über die tote Frau hinweg und bemerkt sie nicht: er ist auch Patient der Einrichtung und Autist.

Aber auch andere Störungsbilder werden aufgegriffen und in teuren Kinoproduktionen "gezeigt", wie bspw. das Tourette-Syndrom in dem Film Crank aus dem Jahr 2006. Desgleichen gibt es inzwischen eine große Anzahl an Büchern über und von Menschen mit Autismus-Spektrum-Stö- rung. Sie alle vermitteln Bilder von Menschen, die eine Beeinträchtigung haben. Diese Personen werden in Szene gesetzt und dargestellt. Daniel Tammet z.B. hat eine Autobiografie verfasst und tritt auch in Dokumentationen auf. Sein Buch trägt den Titel „Elf ist freundlich und fünf ist laut" und gibt ganz konkrete Eindrücke von den Problemen, die er hat, weil er Autist ist, und wie er sie bewältigt.

Filme, Reportagen und Bücher unterhalten die Nutzer, sie vermitteln aber auch Informationen. Manche Medien sind nicht nur ein Gewinn für die Gesellschaft, sondern sind auch mit Risiken verbunden. In diesem Zusammenhang stellt sich die Frage, welches Bild den Leser/innen von Romanen über Menschen mit Autismus-Spektrum-Störung vermittelt wird und wie sehr die Darstellungen an den wissenschaftlichen Kriterien und den Fakten orientiert sind.

\section{Forschungsfragen und Forschungsziel}

In diesem Vorhaben geht es in erster Linie darum zu analysieren, welche relevanten Aspekte sich aus Romanen über Menschen mit AutismusSpektrum-Störung herausfiltern lassen. Als Filter werden vier Kriterien eingesetzt, u.a. die Beschreibung des Autismus und Situationen, in denen darauf hingewiesen wird, dass die betroffene Person mit ihrem Leben zufrieden ist. Die folgenden zwei Unterfragen werden bearbeitet:

- Welches Bild vermitteln Romane von Menschen mit Autismus-Spektrum-Störung dem Leser/der Leserin?

- Welchen Stellenwert können Romane für die Lehrer/innenausbildung haben?

\section{Autismus-Spektrum-Störung}

Die Bezeichnung Autismus wurde zum ersten Mal von dem Schweizer Psychologen Eugen Bleuler (1857-1939) verwendet. Er bezog sich auf Personen mit Schizophrenie, bei denen er Verhaltensweisen von sozialem Rückzug, Flucht in die eigene Gedankenwelt und verringerte kommunikative Fähigkeiten beobachtete (Tebartz van Elst 2013). Autismus ist eine komplexe Beeinträchti- 
gung, welche bis heute nicht komplett entschlüsselt worden ist. Das hängt damit zusammen, dass nicht ganz klar ist, welche Fragen gestellt werden sollen; die Folge davon ist, dass sich der Forschungsprozess als sehr langwierig erweist und es kaum empirisch fundierte Theorien gibt (Shore/Rastelli 2006). Die Autismus-Spektrum-Störung zeigt sich in der Beeinträchtigung von kommunikativen und sozial-interaktiven Fähigkeiten in unterschiedlichen Settings. Nicht nur die gängigen Symptome (Defizite im sozialemotionalen Verhalten, in der nonverbalen Kommunikation und im Aufbau und der Pflege von Beziehungen), sondern auch andere Störungsbilder (z.B. ADHS) gehen mit der autistischen Störung einher (Bowler 2007; American Psychiatric Association 2013). Aus diesem Grund überrascht es nicht, dass in entsprechenden Veröffentlichungen ganz unterschiedliche Merkmale genannt werden. So betonen Wing (1981) und Gilberg und Gilberg (1989) bspw. motorische Beeinträchtigungen und das einnehmende Interesse für ein Spezialgebiet, während Shore und Rastelli (2006) eher die sensorische Überforderung und die Art und Weise, wie Menschen mit Autismus-Spektrum-Störung denken, fokussieren.

\section{Lebenszufriedenheit}

Im Zusammenhang mit der qualitativ hochwertigen und effizienten medizinischen Versorgung von Patienten wurde das Thema Lebenszufriedenheit zum ersten Mal als relevant angesehen (Angermeyer u.a. 2000). Eine Analyse verschiedener wissenschaftlicher Studien zeigt, dass es ganz unterschiedliche Kategorien und Dimensionen gibt, mit denen versucht wird, das Phänomen zu beschreiben (Schalock 2000; Frisch 2006; Diener 2009). Des Weiteren gibt es mehrere Begriffe, die im Sprachgebrauch synonym verwendet und selten trennscharf differenziert werden (Glück, Lebensqualität, Wohlbefinden ...). In der Scientific Community scheint es keinen Konsens darüber zu geben, ob Lebenszufriedenheit eher eine allgemeine und umfassende individuelle Betrachtungsweise des Lebens jeder einzelnen Person ist oder in bestimmte Domänen zusammengefasst werden kann. Es stellt sich die Frage, wie in nicht wissenschaftlichen Veröffentlichungen (hier: Romane) mit der Thematik umgegangen wird und welche Lehren daraus gezogen werden können.

\section{Methodik}

Aus einer Reihe von Literaturvorschlägen wurden fünf Romane ausgewählt und gelesen. Die Vorschläge stammten u.a. von zwei Personen mit Autismus-Spektrum-Störung. Unter den ausgesuchten Büchern waren solche, die von Autoren beiderlei Geschlechts verfasst waren, die selber von Autismus betroffen sind, und Romane, deren Autor neuro-typisch, der Hauptprotagonist der Erzählung dagegen Autist ist. Auf Basis der qualitativen Inhaltsanalyse nach Mayring werden die Romane kategoriengeleitet durchgearbeitet. Dabei wird der Fokus auf zwei der drei, das Auswertungskonzept umfassende Schritte gelegt: Zusammenfassende Inhaltsanalyse und Strukturierende Inhaltsanalyse. Auf die Explizierende Inhaltsanalyse wird in dem Forschungsprojekt verzichtet. Ergänzend dazu wird jedoch die Vorgeschichte der Autorlinnen (und ggfls. auch der Übersetzer/innen) der Bücher näher betrachtet (Bortz/Döring 2006).

Weitere Informationen und Literaturangaben können eingeholt werden bei carmen.feldhaus@ uni-oldenburg.de 